\title{
Idempotent and Regular Elements of the Complete Semigroups of Binary Relations of the Class $\Sigma_{3}(X, 9)$
}

\author{
Barış Albayrak, Neşet Aydın \\ Çanakkale Onsekiz Mart University, Çanakkale, Turkey \\ Email: balbayrak77@gmail.com, neseta@comu.edu.tr
}

Received 13 January 2015; accepted 1 February 2015; published 5 February 2015

Copyright (C) 2015 by authors and Scientific Research Publishing Inc.

This work is licensed under the Creative Commons Attribution International License (CC BY).

http://creativecommons.org/licenses/by/4.0/

cc) (i)

\begin{abstract}
In this paper, we take $Q_{16}$ subsemilattice of $D$ and we will calculate the number of right unit, idempotent and regular elements $\alpha$ of $B_{X}\left(Q_{16}\right)$ satisfied that $V(D, \alpha)=Q_{16}$ for a finite set $X$. Also we will give a formula for calculate idempotent and regular elements of $B_{X}(Q)$ defined by an $X$-semilattice of unions $D$.
\end{abstract}

Keywords

Semilattice, Semigroup, Binary Relation

\section{Introduction}

Let $X$ be a nonempty set and $B_{X}$ be semigroup of all binary relations on the set $X$. If $D$ is a nonempty set of subsets of $X$ which is closed under the union then $D$ is called a complete $X$-semilattice of unions.

Let $f$ be an arbitrary mapping from $X$ into $D$. Then one can construct a binary relation $\alpha_{f}$ on $X$ by $\alpha_{f}=\bigcup_{x \in X}(\{x\} \times f(x))$. The set of all such binary relations is denoted by $B_{X}(D)$ and called a complete semigroup of binary relations defined by an $X$-semilattice of unions $D$.

We use the notations, $y \alpha=\{x \in X \mid y \alpha x\}, Y \alpha=\bigcup_{y \in Y} y \alpha, V(D, \alpha)=\{Y \alpha \mid Y \in D\}, Y_{T}^{\alpha}=\{y \in X \mid y \alpha=T\}$.

A representation of a binary relation $\alpha$ of the form $\alpha=\bigcup_{T \in V\left(X^{*}, \alpha\right)}\left(Y_{T}^{\alpha} \times T\right)$ is called quasinormal. Note that, 
if $\alpha=\bigcup_{T \in V\left(X^{*}, \alpha\right)}\left(Y_{T}^{\alpha} \times T\right)$ is a quasinormal representation of the binary relation $\alpha$, then $Y_{T}^{\alpha} \cap Y_{T^{\prime}}^{\alpha}=\varnothing$ for $T$, $T^{\prime} \in V\left(X^{*}, \alpha\right)$ and $T \neq T^{\prime}$.

A complete $X$-semilattice of unions $D$ is an $X I$-semilattice of unions if $\Lambda\left(D, D_{t}\right) \in D$ for any $t \in \breve{D}$ and $Z=\bigcup_{t \in Z} \Lambda\left(D, D_{t}\right)$ for any nonempty element $Z$ of $D$.

Now, $\alpha \in B_{X}(D)$ is said to be right unit if $\beta \circ \alpha=\beta$ for all $\beta \in B_{X}(D)$. Also, $\alpha \in B_{X}(D)$ is idempotent if $\alpha \circ \alpha=\alpha$. And $\alpha \in B_{X}(D)$ is said to be regular if $\alpha \circ \beta \circ \alpha=\alpha$ for some $\beta \in B_{X}(D)$.

Let $D^{\prime}, D^{\prime \prime}$ be complete $X$-semilattices of unions and $\varphi$ be a one-to-one mapping from $D^{\prime}$ to $D^{\prime \prime}$. A mapping $\varphi: D^{\prime} \rightarrow D^{\prime \prime}$ is a complete isomorphism provided $\varphi\left(\cup D_{1}\right)=\bigcup_{T^{\prime} \in D_{1}} \varphi\left(T^{\prime}\right)$ for all nonempty subset $D_{1}$ of the semilattice $D^{\prime}$. Besides that, if $\varphi: V(D, \alpha) \rightarrow D^{\prime}$ is a complete isomorphism where $\alpha \in B_{X}(D), \varphi(T) \alpha=T$ for all $T \in V(D, \alpha), \varphi$ is said to be a complete $\alpha$-isomorphism.

Let $Q$ and $D^{\prime}$ be respectively some $X I$ and $X$-subsemilattices of the complete $X$-semilattice of unions $D$. Then

$$
R_{\varphi}\left(Q, D^{\prime}\right)=\left\{\alpha \in B_{X}(D) \mid \alpha \text { regular element, } \varphi \text { complete } \alpha \text {-isomorphism }\right\}
$$

where $\varphi: Q \rightarrow D^{\prime}$ complete isomorphism and $V(D, \alpha)=Q$. Besides, let us denote

where

$$
R\left(Q, D^{\prime}\right)=\bigcup_{\varphi \in \Phi\left(Q, D^{\prime}\right)} R_{\varphi}\left(Q, D^{\prime}\right) \text { and } R\left(D^{\prime}\right)=\bigcup_{Q^{\prime} \in \Omega(Q)} R\left(Q^{\prime}, D^{\prime}\right)
$$

$$
\begin{gathered}
\Phi\left(Q, D^{\prime}\right)=\left\{\varphi \mid \varphi: Q \rightarrow D^{\prime} \text { is a complete } \alpha \text {-isomorhism } \exists \alpha \in B_{X}(D)\right\} \\
\Omega(Q)=\left\{Q^{\prime} \mid Q^{\prime} \text { is } X I \text {-subsemilattices of } D \text { which is complete isomorphic to } Q\right\}
\end{gathered}
$$

This structure was comprehensively investigated in Diasamidze [1].

Lemma 1. [1] If $Q$ is complete $X$-semilattice of unions and $I(Q)$ is the set all right units of the semigroup $B_{X}(Q)$ then $I(Q)=R_{i d_{Q}}(Q, Q)$.

Lemma 2. [2] Let $X$ be a finite set, $D$ be a complete $X$-semilattice of unions and $Q=\left\{T_{1}, T_{2}, T_{3}, T_{4}, T_{5}, T_{6}, T_{7}, T_{8}\right\}$ be $X$-subsemilattice of unions of $D$ satisfies the following conditions

$$
\begin{array}{ll}
T_{1} \subset T_{2} \subset T_{3} \subset T_{5} \subset T_{6} \subset T_{8}, & T_{1} \subset T_{2} \subset T_{3} \subset T_{5} \subset T_{7} \subset T_{8}, \\
T_{1} \subset T_{2} \subset T_{4} \subset T_{5} \subset T_{6} \subset T_{8}, & T_{1} \subset T_{2} \subset T_{4} \subset T_{5} \subset T_{7} \subset T_{8}, \\
T_{4} \backslash T_{3} \neq \varnothing, T_{3} \backslash T_{4} \neq \varnothing, & T_{6} \backslash T_{7} \neq \varnothing, T_{7} \backslash T_{6} \neq \varnothing, \\
T_{3} \cup T_{4}=T_{5} T_{6} \cup T_{7}=T_{8} & T_{1} \neq \varnothing .
\end{array}
$$

$Q$ is $X I$-semilattice of unions.

Theorem 1. [2] Let $X$ be a finite set and $Q$ be XI-semilattice. If $D^{\prime}=\left\{\bar{T}_{1}, \bar{T}_{2}, \bar{T}_{3}, \bar{T}_{4}, \bar{T}_{5}, \bar{T}_{6}, \bar{T}_{7}, \bar{T}_{8}\right\}$ is $\alpha$-isomorphic to $Q$ and $\Omega(Q)=m_{0}$, then

$$
\begin{aligned}
\left|R\left(D^{\prime}\right)\right|= & m_{0} \cdot 4 \cdot\left(2^{\left|\left(\bar{T}_{3} \cap \bar{T}_{4}\right) \backslash \bar{T}_{1}\right|}\left(2^{\left|\bar{T}_{2} \backslash \bar{T}_{1}\right|}-1\right)\right) \cdot\left(3^{\left|\bar{T}_{4} \backslash \bar{T}_{3}\right|}-2^{\left|\bar{T}_{4} \backslash \bar{T}_{3}\right|}\right) \cdot\left(3^{\left|\bar{T}_{3} \backslash \bar{T}_{4}\right|}-2^{\left|\overline{T_{3}} \backslash \bar{T}_{4}\right|}\right) \cdot 5^{\left|\left(\bar{T}_{7} \cap \bar{T}_{6}\right) \backslash \bar{T}_{5}\right|} \\
& \cdot\left(6^{\left|\bar{T}_{7} \bar{T}_{6}\right|}-5^{\left|\bar{T}_{7} \backslash \bar{T}_{6}\right|}\right) \cdot\left(6^{\left|\bar{T}_{6} \backslash \bar{T}_{7}\right|}-5^{\left|\bar{T}_{6} \backslash \bar{T}_{7}\right|}\right) \cdot 8^{\left|X \backslash \bar{T}_{8}\right|} \cdot
\end{aligned}
$$

Theorem 2. [2] Let $\alpha \in B_{X}(Q)$ be a quasinormal representation of the form $\alpha=\bigcup_{i=1}^{8}\left(Y_{i}^{\alpha} \times T_{i}\right)$ such that $V(D, \alpha)=Q . \alpha \in B_{X}(D)$ is a regular iff for some complete $\alpha$-isomorphism $\varphi: Q \rightarrow D^{\prime} \subseteq D$, the following conditions are satisfied:

$$
\begin{aligned}
& Y_{1}^{\alpha} \supseteq \varphi\left(T_{1}\right), \quad Y_{1}^{\alpha} \cup Y_{2}^{\alpha} \supseteq \varphi\left(T_{2}\right), \quad Y_{1}^{\alpha} \cup Y_{2}^{\alpha} \cup Y_{3}^{\alpha} \supseteq \varphi\left(T_{3}\right), \\
& Y_{1}^{\alpha} \cup Y_{2}^{\alpha} \cup Y_{4}^{\alpha} \supseteq \varphi\left(T_{4}\right), \quad Y_{1}^{\alpha} \cup Y_{2}^{\alpha} \cup Y_{3}^{\alpha} \cup Y_{4}^{\alpha} \cup Y_{5}^{\alpha} \cup Y_{6}^{\alpha} \supseteq \varphi\left(T_{6}\right), \\
& Y_{1}^{\alpha} \cup Y_{2}^{\alpha} \cup Y_{3}^{\alpha} \cup Y_{4}^{\alpha} \cup Y_{5}^{\alpha} \cup Y_{7}^{\alpha} \supseteq \varphi\left(T_{7}\right), \quad Y_{2}^{\alpha} \cap \varphi\left(T_{2}\right) \neq \varnothing, \\
& Y_{3}^{\alpha} \cap \varphi\left(T_{3}\right) \neq \varnothing, \quad Y_{4}^{\alpha} \cap \varphi\left(T_{4}\right) \neq \varnothing, \quad Y_{6}^{\alpha} \cap \varphi\left(T_{6}\right) \neq \varnothing, \quad Y_{7}^{\alpha} \cap \varphi\left(T_{7}\right) \neq \varnothing .
\end{aligned}
$$


Let $X$ be a finite set and $D=\left\{T_{1}, T_{2}, T_{3}, T_{4}, T_{5}, T_{6}, T_{7}, T_{8}, T_{9}\right\}$ be a complete $X$-semilattice of unions which satisfies the following conditions

$$
\begin{aligned}
& T_{1} \subset T_{3} \subset T_{5} \subset T_{6} \subset T_{8} \subset T_{9}, \\
& T_{1} \subset T_{3} \subset T_{5} \subset T_{6} \subset T_{7} \subset T_{9}, \\
& T_{1} \subset T_{3} \subset T_{4} \subset T_{6} \subset T_{8} \subset T_{9}, \\
& T_{1} \subset T_{3} \subset T_{4} \subset T_{6} \subset T_{7} \subset T_{9}, \\
& T_{2} \subset T_{3} \subset T_{5} \subset T_{6} \subset T_{8} \subset T_{9}, \\
& T_{2} \subset T_{3} \subset T_{5} \subset T_{6} \subset T_{7} \subset T_{9}, \\
& T_{2} \subset T_{3} \subset T_{4} \subset T_{6} \subset T_{8} \subset T_{9}, \\
& T_{2} \subset T_{3} \subset T_{4} \subset T_{6} \subset T_{7} \subset T_{9}, \\
& T_{1} \backslash T_{2} \neq \varnothing, T_{2} \backslash T_{1} \neq \varnothing, T_{4} \backslash T_{5} \neq \varnothing, \\
& T_{5} \backslash T_{4} \neq \varnothing, T_{7} \backslash T_{8} \neq \varnothing, T_{8} \backslash T_{7} \neq \varnothing, \\
& T_{1} \cup T_{2}=T_{3}, T_{4} \cup T_{5}=T_{6}, \\
& T_{7} \cup T_{8}=T_{9}, T_{1} \cap T_{2} \neq \varnothing
\end{aligned}
$$

The diagram of the $D$ is shown in Figure 1. By the symbol $\sum_{3}(X, 9)$ we denote the class of all complete $X$ semilattice of unions whose every element is isomophic to an $X$-semilattice of the form $D$.

All subsemilattice of $D=\left\{T_{1}, T_{2}, T_{3}, T_{4}, T_{5}, T_{6}, T_{7}, T_{8}, T_{9}\right\}$ are given in Figure 2.

In Diasamidze [1], it has shown that subsemilattices 1 - 15 are XI-semilattice of unions and subsemilattices 17 24 are not $X I$-semilattice of unions. In Yeşil Sungur [3] and Albayrak [4], they have shown that subsemilattices 25 and 26 are $X I$-semilattice of unions if and only if $T_{1} \cap T_{2}=\varnothing$ ”. Also they found that number of right unit, idempotent and regular elements in subsemilattices.

In this paper, we take in particular, $Q_{16}=\left\{T, T_{3}, T_{4}, T_{5}, T_{6}, T_{7}, T_{8}, T_{9}\right\}$ subsemilattice of $D$. We will calculate the number of right unit, idempotent and regular elements $\alpha$ of $B_{X}\left(Q_{16}\right)$ satisfied that $V(D, \alpha)=Q_{16}$ for a finite set $X$. Also we will give a formula for calculate idempotent and regular elements of $B_{X}(D)$ defined by an $X$-semilattice of unions $D=\left\{T_{1}, T_{2}, T_{3}, T_{4}, T_{5}, T_{6}, T_{7}, T_{8}, T_{9}\right\}$.

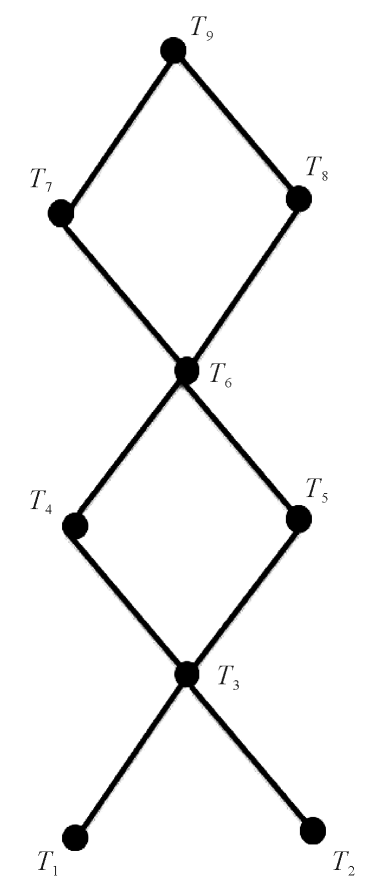

Figure 1. Diagram of $D$. 


\section{Results}

Let $Q_{16}=\left\{T, T_{3}, T_{4}, T_{5}, T_{6}, T_{7}, T_{8}, T_{9}\right\}$ be complete $X$-subsemilattice of $D$ satisfies the following conditions

$$
\begin{gathered}
T \subset T_{3} \subset T_{4} \subset T_{6} \subset T_{7} \subset T_{9}, \\
T \subset T_{3} \subset T_{5} \subset T_{6} \subset T_{7} \subset T_{9}, \\
T \subset T_{3} \subset T_{4} \subset T_{6} \subset T_{8} \subset T_{9}, \\
T \subset T_{3} \subset T_{5} \subset T_{6} \subset T_{8} \subset T_{9}, \\
T_{4} \backslash T_{5} \neq \varnothing, T_{5} \backslash T_{4} \neq \varnothing, \\
T_{7} \backslash T_{8} \neq \varnothing, T_{8} \backslash T_{7} \neq \varnothing, \\
T_{4} \cup T_{5}=T_{6}, T_{8} \cup T_{7}=T_{9} \\
T \neq \varnothing .
\end{gathered}
$$

The diagram of the $Q_{16}$ is shown in Figure 3. From Lemma $2 Q_{16}$ is $X I$-semilattice of unions.

Let $Q_{16} \vartheta_{X I}$ denote the set of all $X I$-subsemilattice of the semilattice $D$ which are isomorphic of the $X$-semilattice $Q_{16}$. Then we get

$$
Q_{16} \vartheta_{X I}=\left\{\left\{T_{1}, T_{3}, T_{4}, T_{5}, T_{6}, T_{7}, T_{8}, T_{9}\right\},\left\{T_{2}, T_{3}, T_{4}, T_{5}, T_{6}, T_{7}, T_{8}, T_{9}\right\}\right\}
$$

Let $\alpha \in B_{X}\left(Q_{16}\right)$ be a idempotent element having a quasinormal representation of the form
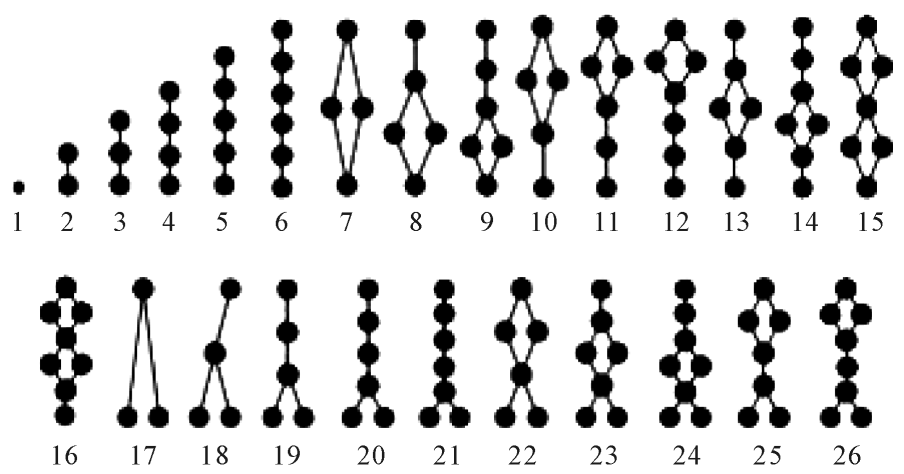

Figure 2. All subsemilattice of $D$.

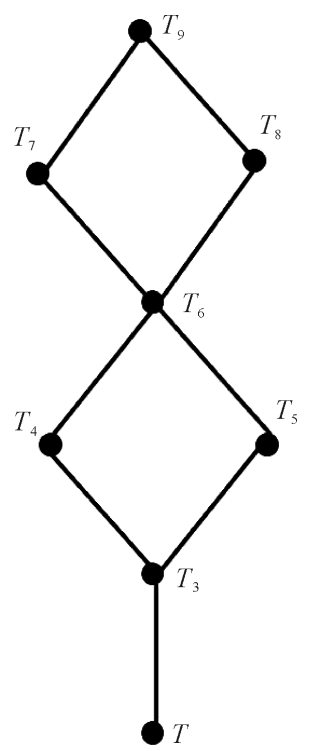

Figure 3. The diagram of the $Q_{16}$. 
$\alpha=\left(Y_{T}^{\alpha} \times T\right) \cup \bigcup_{i=3}^{9}\left(Y_{i}^{\alpha} \times T_{i}\right)$, such that $V(D, \alpha)=Q_{16}$. First we calculate number of this idempotent elements in $B_{X}\left(Q_{16}\right)$.

Lemma 3. If $X$ is a finite set and $I\left(Q_{16}\right)$ is the set all right units of the semigroup $B_{X}\left(Q_{16}\right)$, then the number $\left|I\left(Q_{16}\right)\right|$ may be calculated by formula:

$$
\begin{aligned}
\left|I\left(Q_{16}\right)\right|= & \left(\left(2^{\left|T_{3} \backslash T\right|}-1\right) \cdot 2^{\left|\left(T_{5} \cap T_{4}\right) \backslash T_{3}\right|}\right) \cdot\left(3^{\left|T_{5} \backslash \backslash_{4}\right|}-2^{\left|T_{5} \backslash T_{4}\right|}\right) \cdot\left(3^{\left|T_{4} \backslash T_{5}\right|}-2^{\left|T_{4} \backslash T_{5}\right|}\right) \\
& \cdot 5^{\left|\left(T_{7} \cap T_{8}\right) \backslash T_{6}\right|} \cdot\left(6^{\left|T_{8} \backslash T_{7}\right|}-5^{\left|T_{8} \backslash T_{7}\right|}\right) \cdot\left(6^{\left|T_{7} \backslash T_{8}\right|}-5^{\left|T_{7} \backslash T_{8}\right|}\right) \cdot 8^{\left|X \backslash T_{9}\right|} .
\end{aligned}
$$

Proof. From Lemma 1 we have $I\left(Q_{16}\right)=R_{i d_{Q_{16}}}\left(Q_{16}, Q_{16}\right)$ where $i d_{Q_{16}}$ is identity mapping of the set $Q_{16}$. For this reason $D^{\prime}=Q$ in Theorem 1 . Then we obtain

$$
\begin{aligned}
\left|I\left(Q_{16}\right)\right|= & \left(\left(2^{\left|T_{3} \backslash T\right|}-1\right) \cdot 2^{\left|\left(T_{5} \cap T_{4}\right) \backslash T_{3}\right|}\right) \cdot\left(3^{\left|T_{5} \backslash T_{4}\right|}-2^{\left|T_{5} \backslash T_{4}\right|}\right) \cdot\left(3^{\left|T_{4} \backslash T_{5}\right|}-2^{\left|T_{4} \backslash T_{5}\right|}\right) \\
& \cdot 5^{\left|\left(T_{7} \cap T_{8}\right) \backslash T_{6}\right|} \cdot\left(6^{\left|T_{8} \backslash T_{7}\right|}-5^{\left|T_{8} \backslash T_{7}\right|}\right) \cdot\left(6^{\left|T_{7} \backslash T_{8}\right|}-5^{\left|T_{7} \backslash T_{8}\right|}\right) \cdot 8^{\left|X \backslash T_{9}\right|} .
\end{aligned}
$$

Theorem 3. If $X$ is a finite set and $I^{*}\left(Q_{16}\right)$ is the set all idempotent elements of the semigroup $B_{X}\left(Q_{16}\right)$, then the number $\left|I^{*}\left(Q_{16}\right)\right|$ may be calculated by formula:

$$
\begin{aligned}
\left|I^{*}\left(Q_{16}\right)\right|= & \left(\left(2^{\left|T_{3} \backslash T_{2}\right|}-1\right) \cdot 2^{\left|\left(T_{5} \cap T_{4}\right) \backslash T_{3}\right|}\right) \cdot\left(3^{\left|T_{5} \backslash T_{4}\right|}-2^{\left|T_{5} \backslash T_{4}\right|}\right) \cdot\left(3^{\left|T_{4} \backslash T_{5}\right|}-2^{\left|T_{4} \backslash T_{5}\right|}\right) \\
& \cdot 5^{\left|\left(T_{7} \cap T_{8}\right) \backslash T_{6}\right|} \cdot\left(6^{\left|T_{8} \backslash T_{7}\right|}-5^{\left|T_{8} \backslash T_{7}\right|}\right) \cdot\left(6^{\left|T_{7} \backslash T_{8}\right|}-5^{\left|T_{7} \backslash T_{8}\right|}\right) \cdot 8^{\left|X \backslash T_{9}\right|} \\
& +\left(\left(2^{\left|T_{3} \backslash T_{1}\right|}-1\right) \cdot 2^{\left|\left(T_{5} \cap T_{4}\right) \backslash T_{3}\right|}\right) \cdot\left(3^{\left|T_{5} \backslash T_{4}\right|}-2^{\left|T_{5} \backslash T_{4}\right|}\right) \cdot\left(3^{\left|T_{4} \backslash T_{5}\right|}-2^{\left|T_{4} \backslash T_{5}\right|}\right) \\
& \cdot 5^{\left|\left(T_{7} \cap T_{8}\right) \backslash T_{6}\right|} \cdot\left(6^{\left|T_{8} \backslash T_{7}\right|}-5^{\left|T_{8} \backslash T_{7}\right|}\right) \cdot\left(6^{\left|T_{7} \backslash T_{8}\right|}-5^{\left|T_{7} \backslash T_{8}\right|}\right) \cdot 8^{\left|X \backslash T_{9}\right|}
\end{aligned}
$$

Proof. By using Lemma 3 we have number of right units of the semigroup $B_{X}\left(Q_{16}\right)$ defined by $Q_{16}=\left\{T, T_{3}, T_{4}, T_{5}, T_{6}, T_{7}, T_{8}, T_{9}\right\}$ for $T \in\left\{T_{1}, T_{2}\right\}$. Then number of idempotent elements of $I^{*}\left(Q_{16}\right)$ calculated by formula $I^{*}\left(Q_{16}\right)=\sum_{D^{\prime} \in Q_{16} \vartheta_{X I}}\left|I\left(D^{\prime}\right)\right|$. By using

$$
Q_{16} \vartheta_{X I}=\left\{\left\{T_{1}, T_{3}, T_{4}, T_{5}, T_{6}, T_{7}, T_{8}, T_{9}\right\},\left\{T_{2}, T_{3}, T_{4}, T_{5}, T_{6}, T_{7}, T_{8}, T_{9}\right\}\right\}
$$

we obtain above formula.

Now we will calculate number of regular elements $\alpha \in B_{X}\left(Q_{16}\right)$ having a quasinormal representation of the form $\alpha=\left(Y_{T}^{\alpha} \times T\right) \cup \bigcup_{i=3}^{9}\left(Y_{i}^{\alpha} \times T_{i}\right)$ such that $V(D, \alpha)=Q_{16}$. Let $R^{*}\left(Q_{16}\right)$ be the set all regular elements of the semigroup $B_{X}\left(Q_{16}\right)$. By using $Q_{16} \vartheta_{X I}=\left\{\left\{T_{1}, T_{3}, T_{4}, T_{5}, T_{6}, T_{7}, T_{8}, T_{9}\right\},\left\{T_{2}, T_{3}, T_{4}, T_{5}, T_{6}, T_{7}, T_{8}, T_{9}\right\}\right\}$ we get $\left|\Omega\left(Q_{16}\right)\right|=2$. The number of all automorphisms of the semilattice $Q_{16}$ is $q=4$. These are

$$
\begin{aligned}
& I_{Q}=\left(\begin{array}{lllll}
T & T_{3} T_{4} T_{5} T_{6} T_{7} T_{8} T_{9} \\
T & T_{3} T_{4} T_{5} T_{6} T_{7} T_{8} T_{9}
\end{array}\right) \quad \varphi=\left(\begin{array}{llll}
T & T_{3} T_{4} T_{5} T_{6} T_{7} T_{8} T_{9} \\
T & T_{3} T_{5} T_{4} T_{6} T_{7} T_{8} T_{9}
\end{array}\right) \\
& \theta=\left(\begin{array}{llll}
T & T_{3} T_{4} T_{5} T_{6} T_{7} T_{8} T_{9} \\
T & T_{3} T_{4} T_{5} T_{6} T_{8} T_{7} T_{9}
\end{array}\right) \quad \tau=\left(\begin{array}{llll}
T & T_{3} T_{4} T_{5} T_{6} T_{7} T_{8} T_{9} \\
T & T_{3} T_{5} T_{4} T_{6} T_{8} T_{7} T_{9}
\end{array}\right)
\end{aligned}
$$

Then $\left|\Phi\left(Q_{16}\right)\right|=4$. Also by using

$$
\begin{array}{ll}
D_{1}^{\prime}=\left\{T_{2}, T_{3}, T_{4}, T_{5}, T_{6}, T_{7}, T_{8}, T_{9}\right\}, & D_{2}^{\prime}=\left\{T_{2}, T_{3}, T_{5}, T_{4}, T_{6}, T_{7}, T_{8}, T_{9}\right\} \\
D_{3}^{\prime}=\left\{T_{2}, T_{3}, T_{4}, T_{5}, T_{6}, T_{8}, T_{7}, T_{9}\right\}, & D_{4}^{\prime}=\left\{T_{2}, T_{3}, T_{5}, T_{4}, T_{6}, T_{8}, T_{7}, T_{9}\right\} \\
D_{5}^{\prime}=\left\{T_{1}, T_{3}, T_{4}, T_{5}, T_{6}, T_{7}, T_{8}, T_{9}\right\}, & D_{6}^{\prime}=\left\{T_{1}, T_{3}, T_{5}, T_{4}, T_{6}, T_{7}, T_{8}, T_{9}\right\} \\
D_{7}^{\prime}=\left\{T_{1}, T_{3}, T_{4}, T_{5}, T_{6}, T_{8}, T_{7}, T_{9}\right\}, & D_{8}^{\prime}=\left\{T_{1}, T_{3}, T_{5}, T_{4}, T_{6}, T_{8}, T_{7}, T_{9}\right\}
\end{array}
$$


we get $R^{*}\left(Q_{16}\right)=\bigcup_{i=1}^{8} R\left(D_{i}\right)$.

Theorem 4. If $X$ is a finite set and $R^{*}\left(Q_{16}\right)$ is the set all regular elements of the semigroup $B_{X}\left(Q_{16}\right)$, then the number $\left|R^{*}\left(Q_{16}\right)\right|$ may be calculated by formula:

$$
\begin{aligned}
\left|R^{*}\left(Q_{16}\right)\right|= & \cdot \cdot 2\left(\left(2^{\left|T_{3} \backslash T_{2}\right|}-1\right) \cdot 2^{\left|\left(T_{5} \cap T_{4}\right) \backslash T_{3}\right|}\right) \cdot\left(3^{\left|T_{5} \backslash T_{4}\right|}-2^{\left|T_{5} \backslash T_{4}\right|}\right) \cdot 5^{\left|\left(T_{7} \cap T_{8}\right) \backslash T_{6}\right|} \\
& \cdot\left(3^{\left|T_{4} \backslash T_{5}\right|}-2^{\left|T_{4} \backslash T_{5}\right|}\right) \cdot\left(6^{\left|T_{8} \backslash T_{7}\right|}-5^{\left|T_{8} \backslash T_{7}\right|}\right) \cdot\left(6^{\left|T_{7} \backslash T_{8}\right|}-5^{\left|T_{7} \backslash T_{8}\right|}\right) \cdot 8^{\left|X \backslash T_{9}\right|} \\
& +4 \cdot 2 \cdot\left(\left(2^{\left|T_{3} \backslash T_{1}\right|}-1\right) \cdot 2^{\left|\left(T_{5} \cap T_{4}\right) \backslash T_{3}\right|}\right) \cdot\left(3^{\left|T_{5} \backslash T_{4}\right|}-2^{\left|T_{5} \backslash T_{4}\right|}\right) \cdot 5^{\left|\left(T_{7} \cap T_{8}\right) \backslash T_{6}\right|} \\
& \cdot\left(3^{\left|T_{4} \backslash T_{5}\right|}-2^{\left|T_{4} \backslash T_{5}\right|}\right) \cdot\left(6^{\left|T_{8} \backslash T_{7}\right|}-5^{\left|T_{8} \backslash T_{7}\right|}\right) \cdot\left(6^{\left|T_{7} \backslash T_{8}\right|}-5^{\left|T_{7} \backslash T_{8}\right|}\right) \cdot 8^{\left|X \backslash T_{9}\right|}
\end{aligned}
$$

Proof. To account for the elements that are in $R^{*}\left(Q_{16}\right)$, we first subtract out intersection of $R\left(D_{i}^{\prime}\right)$ 's. Let $\alpha \in R\left(D_{1}^{\prime}\right) \cap R\left(D_{2}^{\prime}\right)$. By using Theorem 2 and $Q_{16}=\left\{T, T_{3}, T_{4}, T_{5}, T_{6}, T_{7}, T_{8}, T_{9}\right\}$

$$
\begin{aligned}
\alpha \in R\left(D_{1}^{\prime}\right) \cap R\left(D_{2}^{\prime}\right) \Rightarrow & \alpha \in R\left(D_{1}^{\prime}\right) \text { and } \alpha \in R\left(D_{2}^{\prime}\right) \\
\Rightarrow & Y_{T}^{\alpha} \supseteq T_{2}, Y_{T}^{\alpha} \cup Y_{3}^{\alpha} \supseteq T_{3}, Y_{T}^{\alpha} \cup Y_{3}^{\alpha} \cup Y_{5}^{\alpha} \supseteq T_{5} \\
& Y_{T}^{\alpha} \cup Y_{3}^{\alpha} \cup Y_{4}^{\alpha} \supseteq T_{4}, Y_{T}^{\alpha} \cup Y_{3}^{\alpha} \cup Y_{4}^{\alpha} \cup Y_{5}^{\alpha} \cup Y_{6}^{\alpha} \cup Y_{8}^{\alpha} \supseteq T_{8}, \\
& Y_{T}^{\alpha} \cup Y_{3}^{\alpha} \cup Y_{4}^{\alpha} \cup Y_{5}^{\alpha} \cup Y_{6}^{\alpha} \cup Y_{7}^{\alpha} \supseteq T_{7}, Y_{3}^{\alpha} \cap T_{3} \neq \varnothing, \\
& Y_{4}^{\alpha} \cap T_{4} \neq \varnothing, Y_{5}^{\alpha} \cap T_{5} \neq \varnothing, Y_{7}^{\alpha} \cap T_{7} \neq \varnothing, Y_{8}^{\alpha} \cap T_{8} \neq \varnothing, \\
& Y_{T}^{\alpha} \supseteq T_{2}, Y_{T}^{\alpha} \cup Y_{3}^{\alpha} \supseteq T_{3}, Y_{T}^{\alpha} \cup Y_{3}^{\alpha} \cup Y_{5}^{\alpha} \supseteq T_{4}, \\
& Y_{T}^{\alpha} \cup Y_{3}^{\alpha} \cup Y_{4}^{\alpha} \supseteq T_{5}, Y_{T}^{\alpha} \cup Y_{3}^{\alpha} \cup Y_{4}^{\alpha} \cup Y_{5}^{\alpha} \cup Y_{6}^{\alpha} \cup Y_{8}^{\alpha} \supseteq T_{8}, \\
& Y_{T}^{\alpha} \cup Y_{3}^{\alpha} \cup Y_{4}^{\alpha} \cup Y_{5}^{\alpha} \cup Y_{6}^{\alpha} \cup Y_{7}^{\alpha} \supseteq T_{7}, Y_{3}^{\alpha} \cap T_{3} \neq \varnothing, \\
& Y_{5}^{\alpha} \cap T_{4} \neq \varnothing, Y_{4}^{\alpha} \cap T_{5} \neq \varnothing, Y_{7}^{\alpha} \cap T_{7} \neq \varnothing, Y_{8}^{\alpha} \cap T_{8} \neq \varnothing .
\end{aligned}
$$

We get $\varnothing \neq Y_{4}^{\alpha} \cap T_{4} \subseteq Y_{4}^{\alpha} \cap\left(Y_{T}^{\alpha} \cup Y_{3}^{\alpha} \cup Y_{5}^{\alpha}\right)$ which is a contradiction with $Y_{4}^{\alpha}, Y_{T}^{\alpha}, Y_{3}^{\alpha}, Y_{5}^{\alpha}$ are disjoint sets. Then $R\left(D_{1}^{\prime}\right) \cap R\left(D_{2}^{\prime}\right)=\varnothing$. Smilarly $R\left(D_{i}^{\prime}\right) \cap R\left(D_{j}^{\prime}\right)=\varnothing$ for $i, j=1, \cdots, 6$. Thus we obtain

$$
\left|R^{*}\left(Q_{16}\right)\right|=\left|R\left(D_{1}^{\prime}\right)\right|+\left|R\left(D_{2}^{\prime}\right)\right|+\left|R\left(D_{3}^{\prime}\right)\right|+\left|R\left(D_{4}^{\prime}\right)\right|+\left|R\left(D_{5}^{\prime}\right)\right|+\left|R\left(D_{6}^{\prime}\right)\right|+\left|R\left(D_{7}^{\prime}\right)\right|+\left|R\left(D_{8}^{\prime}\right)\right|
$$

From Theorem 1 we get above formula.

Corollary 1. If $X$ is a finite set, $I_{D}$ is the set all idempotent elements of the semigroup $B_{X}(D)$ and $R_{D}$ is the set all regular elements of the semigroup $B_{X}(D)$, then the number $\left|I_{D}\right|$ and $\left|R_{D}\right|$ may be calculated by formula:

$$
\left|\left(I_{D}\right)\right|=\sum_{i=1}^{16}\left|I^{*}\left(Q_{i}\right)\right|, \quad\left|\left(R_{D}\right)\right|=\sum_{i=1}^{16}\left|R^{*}\left(Q_{i}\right)\right|
$$

Proof. Let $I_{D}$ be the set of all idempotent elements of the semigroup $B_{X}(D)$. Then number of idempotent element of $B_{X}(D)$ is equal to sum of idempotent elements of the subsemigroup defined by $X I$-subsemilattice of $D$. $\left|I^{*}\left(Q_{i}\right)\right|$ is given in Diasamidze [1] for $(i=1,2, \cdots, 15)$. From Theorem 3 we have number of idempotent elements of the subsemigroup $B_{X}\left(Q_{16}\right)$. Then the number $\left|I_{D}\right|$ may be calculated by formula $\left|\left(I_{D}\right)\right|=\sum_{i=1}^{16}\left|I^{*}\left(Q_{i}\right)\right|$. Similarly the number $\left|R_{D}\right|$ may be calculated by formula $\left|\left(R_{D}\right)\right|=\sum_{i=1}^{16}\left|R^{*}\left(Q_{i}\right)\right|$.

\section{References}

[1] Diasamidze, Ya. and Makharadze, Sh. (2013) Complete Semigroups of Binary Relations. Kriter Yayınevi, İstanbul, $524 \mathrm{p}$.

[2] Albayrak, B., Aydin, N. and Diasamidze, Ya. (2013) Reguler Elements of the Complete Semigroups of Binary Relations of the Class $\sum_{7}(X, 8)$. International Journal of Pure and Applied Mathematics, 86, 199-216. http://dx.doi.org/10.12732/ijpam.v86i1.13 
[3] Yeşil Sungur, D. and Aydin, N. (2014) Reguler Elements of the Complete Semigroups of Binary Relations of the Class $\sum_{8}(X, 7)$. General Mathematics Notes, 21, 27-42.

[4] Albayrak, B., Aydin, N. and Yeşil Sungur, D. (2014) Regular Elements of Semigroups $B_{X}(D)$ Defined by the Generalized X-Semilattice. General Mathematics Notes, 23, 96-107. 
Scientific Research Publishing (SCIRP) is one of the largest Open Access journal publishers. It is currently publishing more than 200 open access, online, peer-reviewed journals covering a wide range of academic disciplines. SCIRP serves the worldwide academic communities and contributes to the progress and application of science with its publication.

Other selected journals from SCIRP are listed as below. Submit your manuscript to us via either submit@scirp.org or Online Submission Portal.
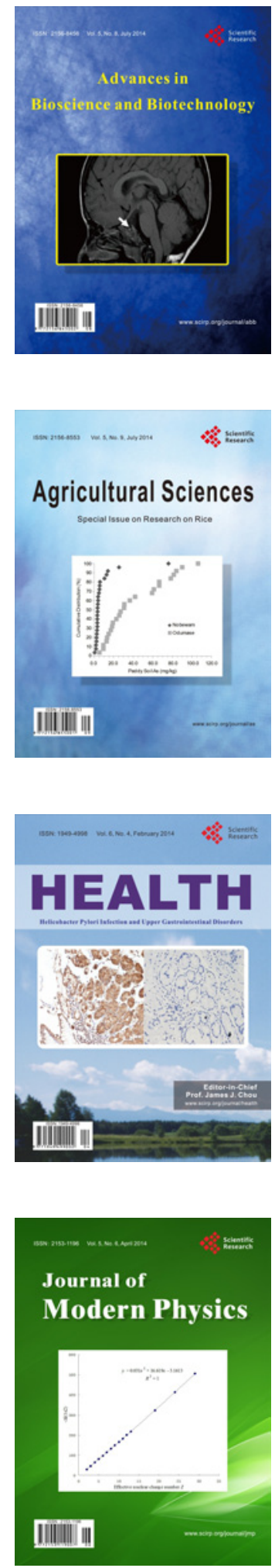
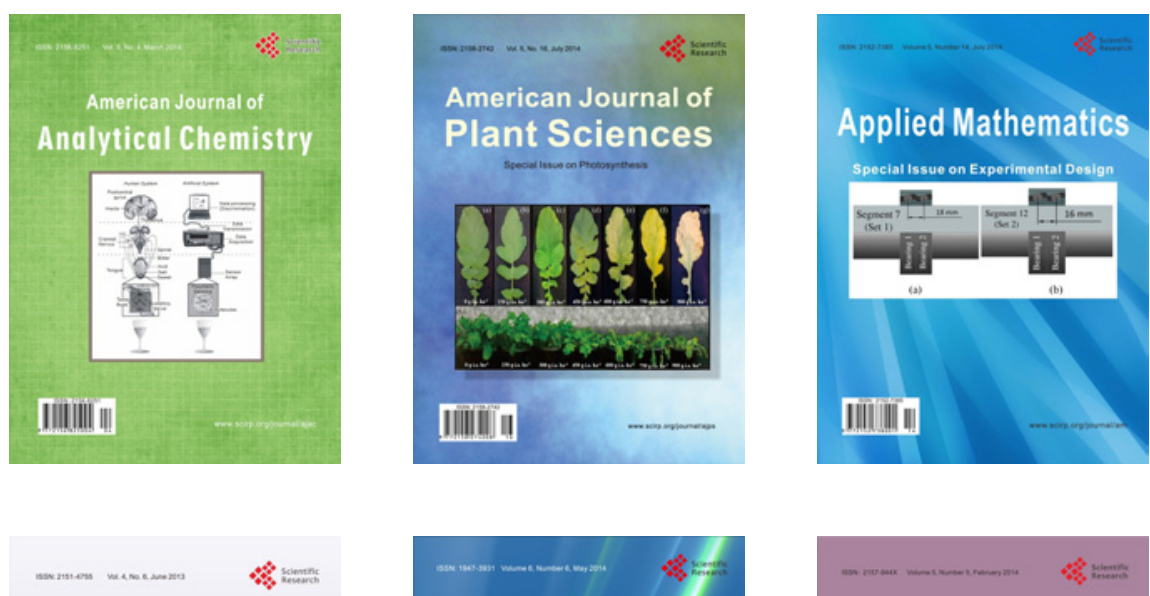

Creative Education
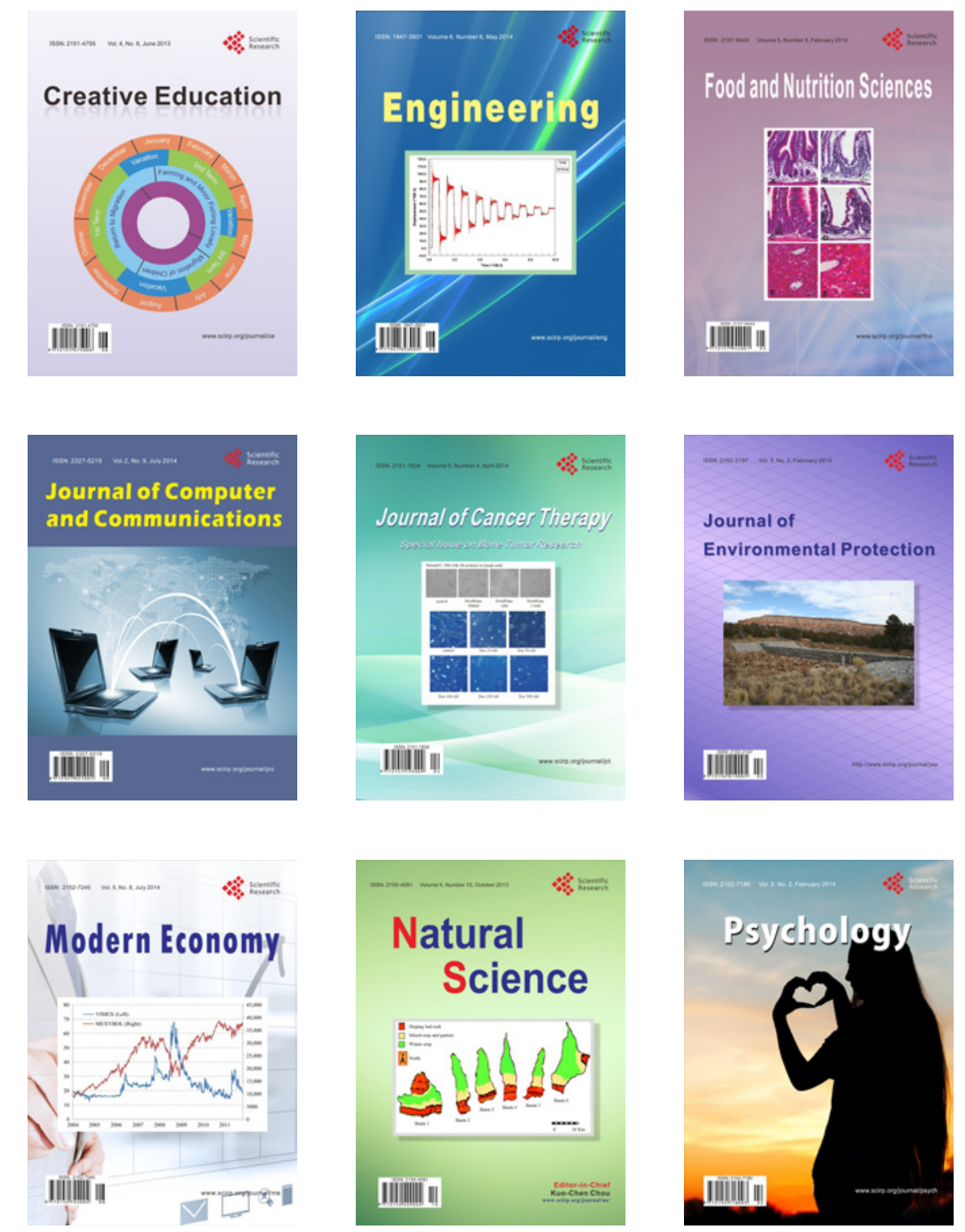